\title{
USING MAGNETIC RESONANCE IMAGING AND PETROGRAPHIC TECHNIQUES TO UNDERSTAND THE TEXTURAL ATTRIBUTES AND POROSITY DISTRIBUTION IN MACARONICHNUS-BURROWED SANDSTONE
}

\author{
MURRAY K. GINGRAS ${ }^{1}$, BRYCE MACMILLAN ${ }^{2}$, BRUCE J. BALCOM², TOM SAUNDERS ${ }^{3}$, AND S. GEORGE PEMBERTON ${ }^{3}$ \\ ${ }^{1}$ Department of Geology, University of New Brunswick, Fredericton, New Brunswick, E3B 5A3, Canada \\ ${ }^{2}$ Department of Physics, MRI Center, University of New Brunswick, Fredericton, New Brunswick, E3B 5A3, Canada \\ ${ }^{3}$ Department of Earth and Atmospheric Sciences, University of Alberta, Edmonton, Alberta, T6G 2E3, Canada \\ e-mail: mgingras@unb.ca
}

\begin{abstract}
Magnetic resonance images are paired with petrographic data to evaluate the textural characteristics of rocks dominated by Macaronichnus segregatis, a trace fossil that is commonly associated with rocks deposited in shallow, marginal marine sedimentary environments. MRI techniques used revealed the three-dimensional geometry of the trace fossil. Burrows are typically horizontal and in plan view range between straight, sinuous, meandering, and spiral geometries. Changes in burrow morphology may be related to population density and patchy resource distribution. The pairing of MRI and petrographic data helped map the distribution of porosity in the burrowed rock. Because MRI images represent complex composites of nuclear spin density and MR relaxation times, each of which is related to pore size, stronger MR signals must be calibrated to known porous zones by integrating petrographic data with MR data. The complex distribution of porosity and its relationship to the matrix show that this fabric represents a dual porosity-permeability system and may affect the resource (reservoir or aquifer) quality of similarly burrowed sedimentary rocks. Future research should elaborate upon the porosity-permeability model for this and similar fabrics.
\end{abstract}

\section{INTRODUCTION}

Sedimentologists use fabric, lithologic contrast, and the presence of diagenetic cements to identify physical and biogenic sedimentary structures in sedimentary rocks. Accordingly, observations that can be made pertaining to rock fabric are limited by the quality of rock exposure or sample available. Importantly, outcrops seldom proffer a three-dimensional understanding of their structure. Nondestructive methods used to resolve the three-dimensional nature (henceforth 3-D) of sedimentary structures at the hand-sample scale include X-ray and X-ray computed tomography (CT). X-rays provide only "flattened"' 3 -D data onto a plane. CT-scans are 3-D, but they are not particularly sensitive to the slight variations in density that characterize most rocks (X-ray devices suffer from the same limitation). Unlike the aforementioned radiographic techniques, magnetic resonance imaging (MRI) does not measure the density of a rock directly; rather it is sensitive to fluids imbibed into the pore space of a rock. Therefore, MRI allows for the 3-D mapping of a magnetic resonance signal, providing a tool that geologists can use to map the porespace distribution in rocks (see "Theory and Methods" section below).

This paper focuses on three main topics: (1) using MRI to image the 3-D nature of Macaronichnus segregatis, the biogenic sedimentary structure used to demonstrate the potential of this method; (2) the paleontological significance of the burrow interrelationships; and, (3) the fluid-flow implications of the textural heterogeneity reported herein.

\section{The Trace Fossil Macaronichnus segregatis}

Clifton and Thompson (1978) first reported the trace fossil Macaronichnus. Since its initial identification, Macaronichnus has proven to be a common trace fossil in Mesozoic and Cenozoic sedimentary rocks worldwide (Saunders and Pemberton 1986; MacEachern and Pemberton 1992; Nara 1994; Gingras et al. 1997). Although the diagnostic taxonomic characteristics remain unpublished, Clifton and Thompson (1978) describe the trace fossil succinctly. In general, it consists of tubular, unbranching burrows that are defined by a grain halo that is lithologically distinct from the surrounding matrix and from the fecal core that fills the trace fossil. Grain-size selection is generally minimal. The burrows are dominantly concordant with laminae, although movement between laminae, and centimeter-scale vertical shifting has been observed at certain localities.

Macaronichnus is an ideal biogenic structure to be resolved three-dimensionally, for several reasons: (1) the burrow morphologies and interrelationships are potentially a taxonomic character; (2) minimal density contrast between the burrow fill and the matrix limits the potential effectiveness of other non destructive methodol- ogies, including X-ray and CT-scan; and, (3) mineralogy aside, the trace fossil is characterized by rather subtle textural changes that result in a relatively homogeneous porosity distribution and will therefore test the potential of MRI as a tool to investigate rock properties.

The analysis presented herein focuses on a sample of Macaronichnus segregatis collected from a Cretaceous outcrop (Horseshoe Canyon Fm., Albian) near Drumheller, Alberta, Canada (Fig. 1). Previous researchers (Saunders and Pemberton 1986) have interpreted the depositional setting of these rocks as a complex composite of marginal marine environments. In the area, horizons characterized by $\mathrm{Ma}$ caronichnus typically cap sedimentary successions and are thought to have developed in the foreshore zone of ancient shorefaces (Saunders and Pemberton 1986).

\section{Previous Work and Methods}

The fundamental difference between X-ray, radiographic, CT, and MRI is the parameters the techniques are capable of imaging. $\mathrm{X}$-ray and gamma-ray techniques are sensitive to electron density. In contrast, MRI images reflect nuclear spin density, MR relaxation times, pore size, and fluid-flow velocities. Unique among the methods listed above, MRI scans can be acquired such that one or more of the aforementioned sample features are highlighted. In porous medium that is characterized by two or more phases, an MRI composite represents a complex interaction between the solid matrix and the fluid- or gas-containing pore space. Nevertheless, MRI has been successfully employed for a variety of petrophysical applications. Examples of these applications include imaging irreducible water in cored reservoir samples (Attard et al. 1994), pore-size distribution and wettability in sedimentary rocks (Howard and Kenyon 1992; Howard 1998), and characterizing fluid movement through solids (Guilfoyle et al. 1996; Bencsik and Ramanathan 2001). Also, nuclear magnetic resonance (NMR) logging has become an important subsurface analysis technique. NMR logging generally has focused on discriminating water, brine, and hydrocarbons in subsurface rocks (Kenyon 1992). More recently it has shown potential in determining the reservoir quality of clay-rich sandstone reservoirs.

Apart from a few applications (cf. Rack et al. 1998) very little work has been initiated that focuses on the problem of resolving physical or biogenic sedimentary structures. This is because the attenuation of MR signals in many materials limits the image quality. To improve image procurement, the sandstone sample was subjected to MRI through stages of progressive desiccation.

Few MRI concepts are commonly applied in sedimentary geology. For this reason the reader is referred to general MRI handbooks that explain the fundamentals of MR image procurement. For example, Matwiyoff (1990) provides an excellent handbook of the basics of MRI technology. Brown and Semelka (1999) have recently published a summary that is useful, but their book is more technical and emphasizes medical applications. The following is a brief summary of the salient details of the MR methodology used in this study. Appendix 1 contains technical details that may be useful if similar studies are conducted. The MR methods applied in this study are related to those employed by Rack et al. (1998).

MR images were generated using conventional spin echo techniques with a comparatively short echo time, such that weak and short-lived signals are better observed. MR images were obtained from a $7 \mathrm{~cm} \times 4 \mathrm{~cm} \times 3 \mathrm{~cm}$ block of burrowed sandstone. A final data matrix consisting of $128^{3}$ data points was produced for the sample. The acquisition time was 55 minutes. The image signal to noise ratio was 16 and the image contrast to noise ratio was 9 . The resolution of each point is approximately 470 microns.

Spin-echo measurements were conducted at ambient temperature with a General Electric (GE NMR Instruments, Fremont, California) CSI II spectrometer in an Oxford (Oxford Instruments, Oxford, England) 2.0 Tesla, $31 \mathrm{~cm}$ i.d. horizontal bore superconducting magnet.

The petrographic analysis consisted of hand-sample and thin-section descriptions. Porosity assessments were made with visual analysis of thin sections of the rock impregnated with blue epoxy. The slides were scanned (digitized) in color using a 


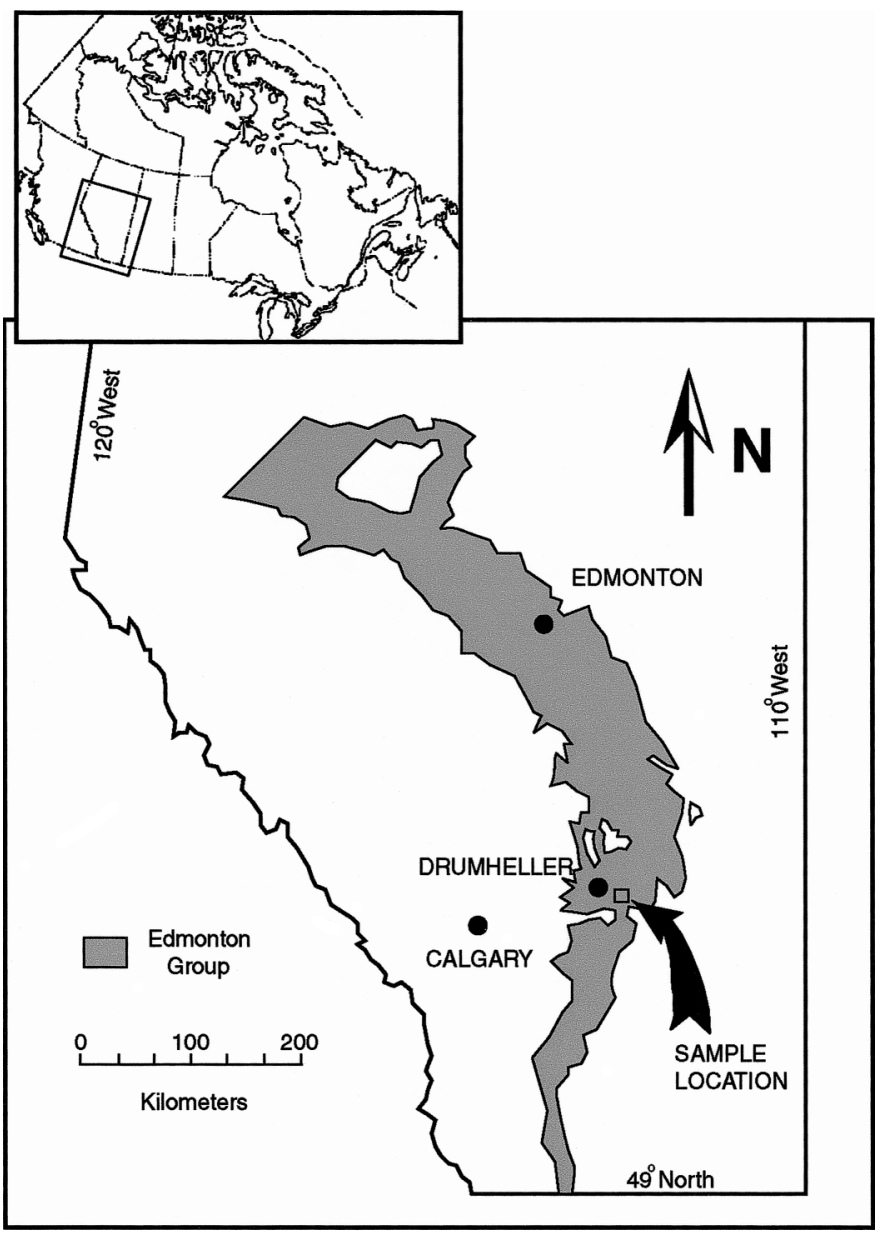

FIG. 1.-Location map. The sample was taken from outcrop near Drumheller, Alberta, Canada. Although the Edmonton Group crops out extensively in Alberta, well-preserved shoreface successions are observed mostly in the area immediately east of Drumheller. Modified from Lavigne (1999).

flatbed scanner. The proportion of blue epoxy was assessed by simple image analysis; the number of blue pixels versus other pixels is the estimated porosity reported forthwith. Because epoxy resin does not necessarily invade the smallest pore spaces, reported porosities may be underestimated by as much as $0.5 \%$. Error was estimated by assessing the void space that was not occupied by the resin.

\section{OBSERVATIONS}

\section{Petrographic Observations}

The sample analyzed is composed of lower-medium-grained sand. Quartz (55\%), plagioclase (25\%), and rock fragments (mostly chert; 15 to 20\%) are present (Fig. $2 \mathrm{~A})$, with minor quantities of mica and volcanic rock fragments. Quartz and chert grains have a diameter close to $2 \phi$. Plagioclase, mica, and lithic grains have more variable sizes, between 1.6 and $2.5 \phi$. Thereby, the comparatively lithic, unburrowed sediment is moderately sorted and the cleaner burrow fills are relatively well sorted.

Grains are subrounded to subangular, and the rock is classified as a texturally submature arkosic arenite (after Dott 1964). Calcite cement has weakly bound the grains, and later siderite cement locally occludes void space (Fig. 2A). The sideritized zones are bedding parallel and are restricted to centimeter- to decimeter-thick horizons.

Because the sample is highly bioturbated, the original laminated character of the rock is no longer evident. However, the pervasive biogenic reworking is primarily bedding parallel and crude bed contacts are still discernible, a common manifestation of Macaronichnus-burrowed media. The grains are not randomly distributed. Burrow cores contain quartz, rare chert, and very rare feldspar. These are surrounded by a halo of chert- and plagioclase-rich sediment (Fig. 2A D). Cross sections of ichnofossils reveal that elongate grains within the burrow are crudely tangentially aligned, whereas the grains in unburrowed sand are apparently unoriented (Fig. 2B). In longitudinal section, elongate grains are arranged orthogonal to the long burrow axis (Fig. 2C). Smaller, more equant grains are situated near the burrow margin (Fig. $2 \mathrm{C})$.

Burrow diameters are clustered around $1.4 \mathrm{~mm}$, which is approximately the mean. The smallest diameter observed is $1.1 \mathrm{~mm}$ and the largest is $1.7 \mathrm{~mm}$, as indicated by the smallest dimension in oblique burrow sections. The ichnofossils are sinuous to meandering and unbranched. Locally curved sections, which may represent spiral segments, are observed. The burrows are generally bedding parallel, but they locally ramp gently upwards or downwards to different horizons; these adjustments seldom exceed one centimeter in a vertical range. Although the sediment is almost completely bioturbated, burrow interpenetrations are not observed. In fact, the burrows only impinge on each other such that their halos coalesce. True cross cutting has not been observed in this material.

Horizons that are only calcite-cemented have 10 to $14 \%$ porosity. Secondary siderite cement reduces the porosity to less than $10 \%$. Sideritized sandstone is separated from calcite-cemented sand by a sharp, well-defined, diagenetic front (white arrow in Fig. 2D). In thin section the porosity in the burrows appears slightly higher than that of the unburrowed sand. Four scanned estimates from longitudinal burrow sections in the calcite-cemented zone were $12.1,13.5,13.8$, and $14.4 \%$ porosity (all porosity measurements are $\pm 0.5 \%$ ). Four porosity estimates from approximately circular burrow cross sections in the calcite-cemented zone were 11.8, 12.8, 13.0, and $13.1 \%$. Porosities of unburrowed sandstone in the calcite zone were 9.9, 10.1, $10.6,11.3,12.4$, and $13.9 \%$. These porosities are lower because of poorer sorting and higher grain-to-grain surface contact in the matrix (Fig. 2A).

\section{MR IMAGES}

Figures 3 and 4 are MR images from the sample of bioturbated sand. Each plate shows selected slices from the 128 available in each dimension. The MRI technique delineates the lower-porosity sideritized zone (black arrows in Fig. 3A) and the higher-porosity, tubular conduits formed by the burrows. Although discrete burrows are relatively well defined, individual grains or pores are not evident. It is notable that the highest MR signals are generated from areas in the rock that are known to have the highest porosities. Because the images represent maps of the distribution of residual water as the rock dries, absolute porosity cannot necessarily be assigned to the image signals. Rather, the images represent differential drainage of burrowed versus unburrowed media, which is in part related to the porosity distribution in the rock. The distribution of MR signals in vertical section (Fig. 3) show that gravity drainage is not a major consideration and that desiccation of the sample occurred mostly through evaporation.

The MRI sections effectively illustrate some of the simple morphologic characteristics of these Macaronichnus. In vertical cross section (Fig. 3) several straight burrows are observable (Fig. 3A-C). Some of the plates show vertical adjustment to adjacent laminae (Fig. 3D; arrow), and deviation from a horizon over a preexisting burrow (Fig. 3E; arrow). As noted from the hand sample, branching is not present. An exception to this may be imaged in Figure 3E, but the apparent branch there could also be explained as a composite of two out-of-plane burrows, which is expected inasmuch as each image slice represents a section of the medium approximately $0.5 \mathrm{~mm}$ thick. Plan-view images demonstrate more clearly the sinuous nature of the burrows (Fig. 4D, E; white arrows). Meander spacings are approximately 1 to 2 centimeters with amplitudes of 0.5 to $2 \mathrm{~cm}$. Meandering segments are discontinuous, breaking into straighter portions or tight proto-spirals in an unpredictable manner. Tight meanders that might be part of spiral activity are rare and poorly developed (Fig. 4A). Furthermore, these features tend to be concentrated in particular zones. Where a meander burrow is evident, a different but similarly curved (meander or partial spiral) trace fossil is commonly observed nearby (Fig. 4D; black arrows). Interpenetrations are rare; a possible example is shown in Figure 4F. These apparent cross cutting relationships may represent out-of-plane burrows that are juxtaposed in an image slice.

Figure 5 is a three-dimensional surface rendering of the MRI signal. The surface represents a transition from approximately $12 \%$ porosity, judging from correlation to petrographic data. The sinuous and interwoven nature of the burrow fabric is apparent; interpenetrations are not evident in the rendering (Fig. 5).

\section{INTERPRETATION AND DISCUSSION}

\section{Interpretation}

Locally common feldspar, and the presence of abundant subrounded to subangular grains indicate that the sediment is submature. Macaronichnus-bearing beds in the Horseshoe Canyon Formation generally cap coarsening-upwards successions. These have been interpreted previously to be shoreface deposits (Saunders and Pemberton, 1986). This is consistent with the presence of Macaronichnus, which has been re- 

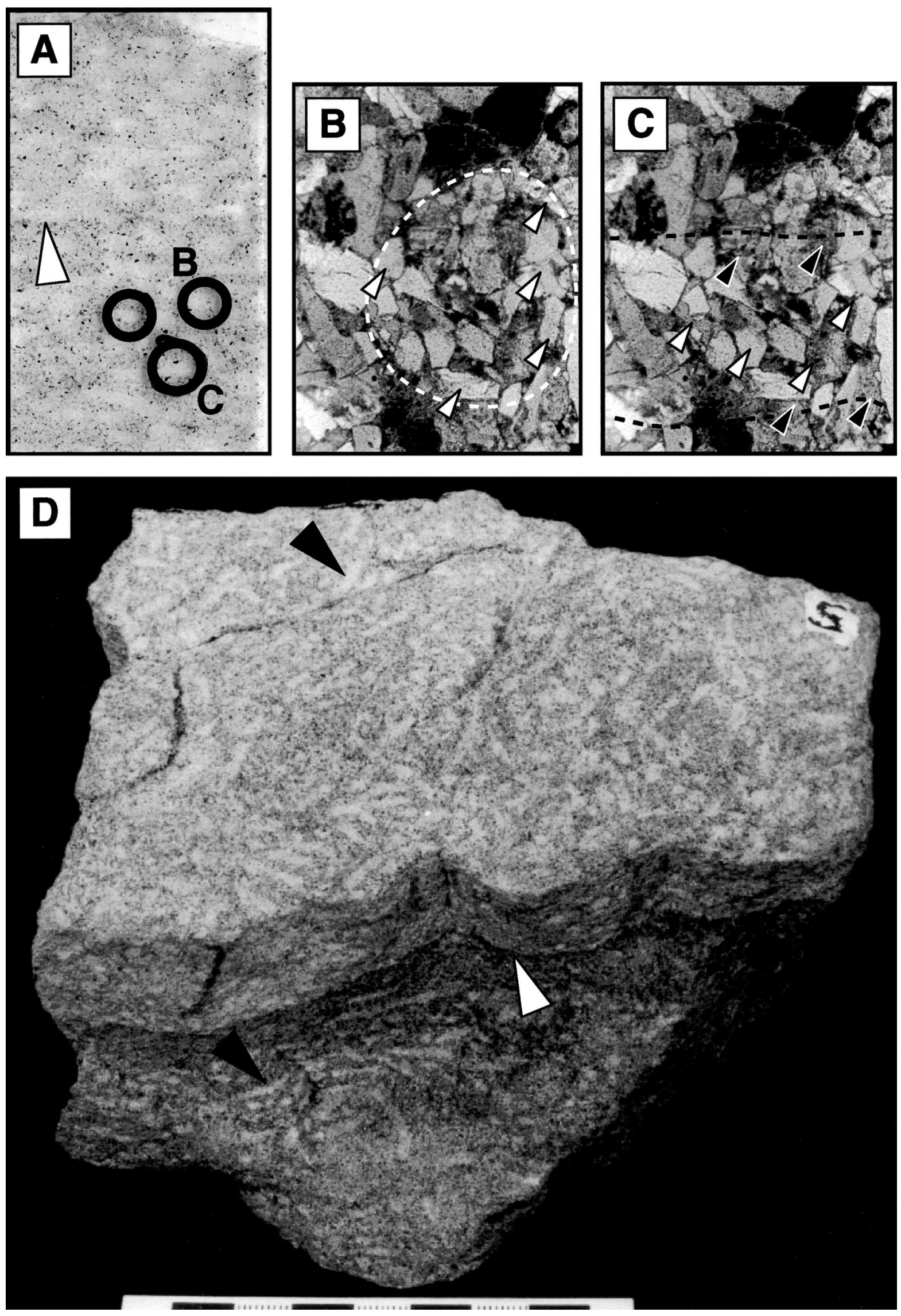

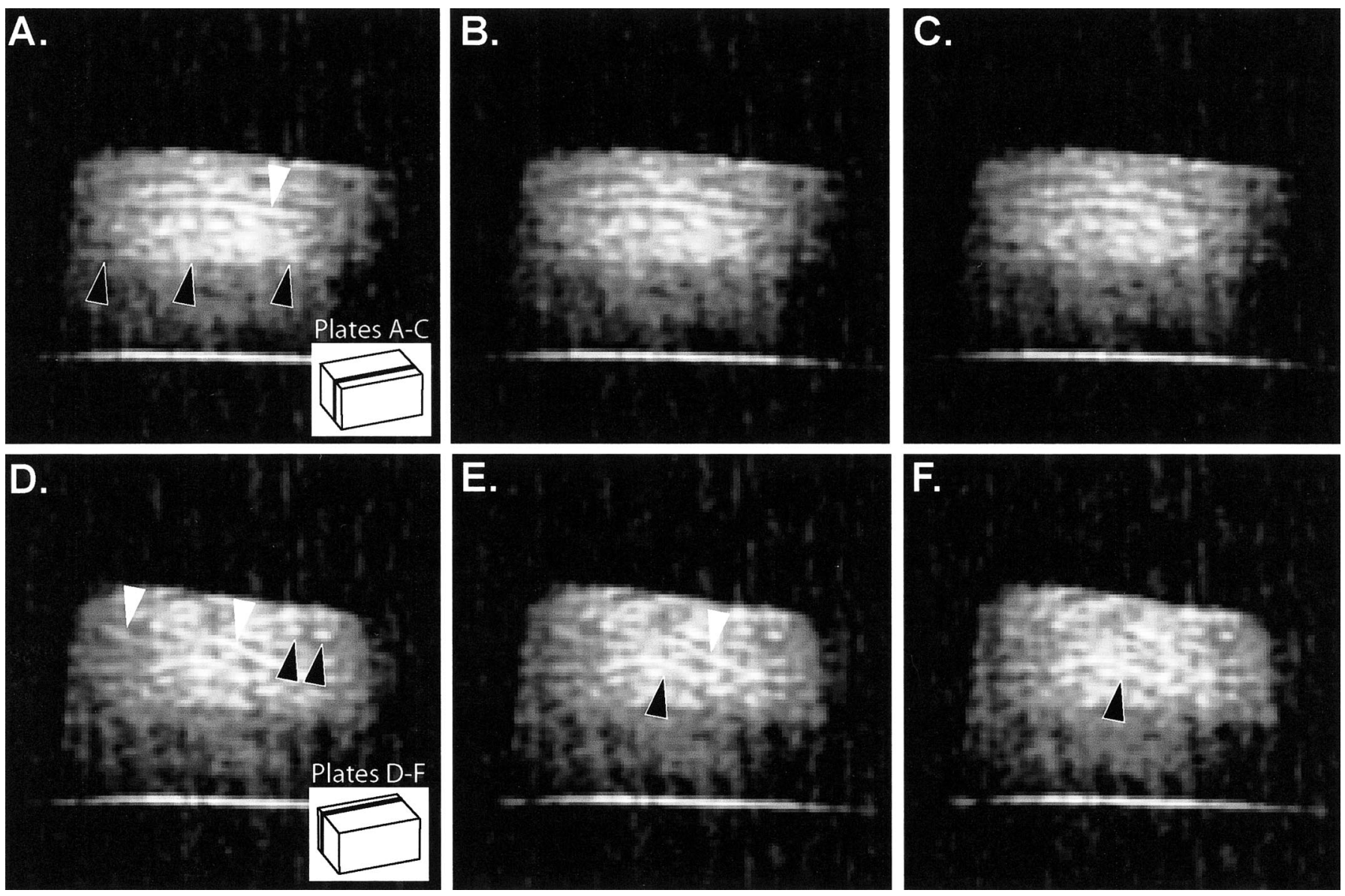

FIG. 3.-MRI elevation views that show a range of behaviors executed by the tracemaking organism. A-C) These frames show primarily horizontal burrows (white arrow), and the contact with the siderite cemented zone (black arrows). Vertical adjustments are visible locally (especially in Part B). The white flash at the bottom of the sample is water-moist cardboard. D-F) These images detail different behaviors of the tracemaking organism as represented by the trace fossil. Namely vertical migration of the animal between laminae is pronounced (white arrows in parts D and E), and avoidance of pre-existing burrows by vertical adjustment is shown (black arrows in parts E and F). Also, a typical side view of truncated burrow meanders (paired bright spots) are evident in Part D (black arrows). An apparent branch is visible in Part F (black arrow), whereas it is seen in Part E that the burrows are separate entities that do not interpenetrate.

ported only from marginal marine accumulations. Texturally, the sediments are less mature than typical shoreline rocks and were likely derived from a nearby point source. From other sedimentological data, including the sedimentary succession, ichnology, and mapped geometry of the deposits, Saunders and Pemberton (1986) and Lavigne (1999) provide a similar interpretation.

The peculiar arrangement of grains within the burrow fill is best explained by studying the burrowing activities of the worm. Tangentially aligned grains probably result from intrusion of the sediment and compactional backfilling of the burrow. Elongate grains aligned perpendicular to the burrow axis are probably the result of the back-and-forth movement of the posterior of the tracemaker as it defecated a quartz-rich fecal cast. This movement was captured on videotape while observing the movements of Euzonis, an opheliid worm known to generate modern Macaronichnus-like traces on the west coast of Canada (Saunders and Pemberton 1990). These movements are documented in Figure 6 A-D. Smaller, equant grains near the burrow margin are swept there by the motion of the worm's posterior. Also, Figure $6 \mathrm{G}-\mathrm{H}$ show the worm Euzonis burrowing against aquarium glass and details of the worm itself.
No satisfactory explanation of the worm's mechanical segregation of chert and feldspar from quartz, all of which have similar specific gravities, has been put forward. The observed enrichment and depletion of the grain fractions is, however, almost certainly the result of grain selection by the trace-making organism. Unfortunately, the question as to how the worm selects a particular mineralogy, and why it does so, is yet to be addressed in the literature. Two possibilities are: (1) the worm can feel and reject angular grains that have pronounced cleavage planes (like feldspar), thus sparing its intestinal tract from sharp grain fragments, and (2) the worm can actually identify some geochemical or biological trace on mineral surfaces and thereby preferentially feeds on microscopic epibionts that preferentially colonize felsic versus mafic grains. Both of these speculations require further research to confirm or discredit them.

Videos of the worm burrowing show only that Euzonis purposefully probes into the sediment with its proboscis and that its chosen direction is not random. Miner et al. (1999) have shown that opheliid larvae are unique among annelids in that they have the ability to feed both with ciliary bands and with their ciliated mouth. Al-

FIG. 2.-Petrographic details and examples of Macaronichnus-burrowed rock taken from outcrop. A) Thin section taken from the imaged sample. Trace fossils (white arrow) appear to be clear because of their quartz-rich fill. B) Cross-sectional view of the trace fossil. White arrows detail the crude tangential arrangement of grains in the burrow fill. A dashed white line delineates the edge of the burrow. C) Longitudinal close-up of a Macaronichnus. In this view, it is seen that grains are arranged perpendicularly to the burrow axis (white arrows), possibly a result of the back-filling behavior of the trace-maker. Smaller grains are concentrated near the edge of the burrow (black arrows). The dashed black line delineates the edge of the burrow. D) Sample of Macaronichnus-burrowed sandstone from outcrop near Drumheller, Alberta, Canada. 

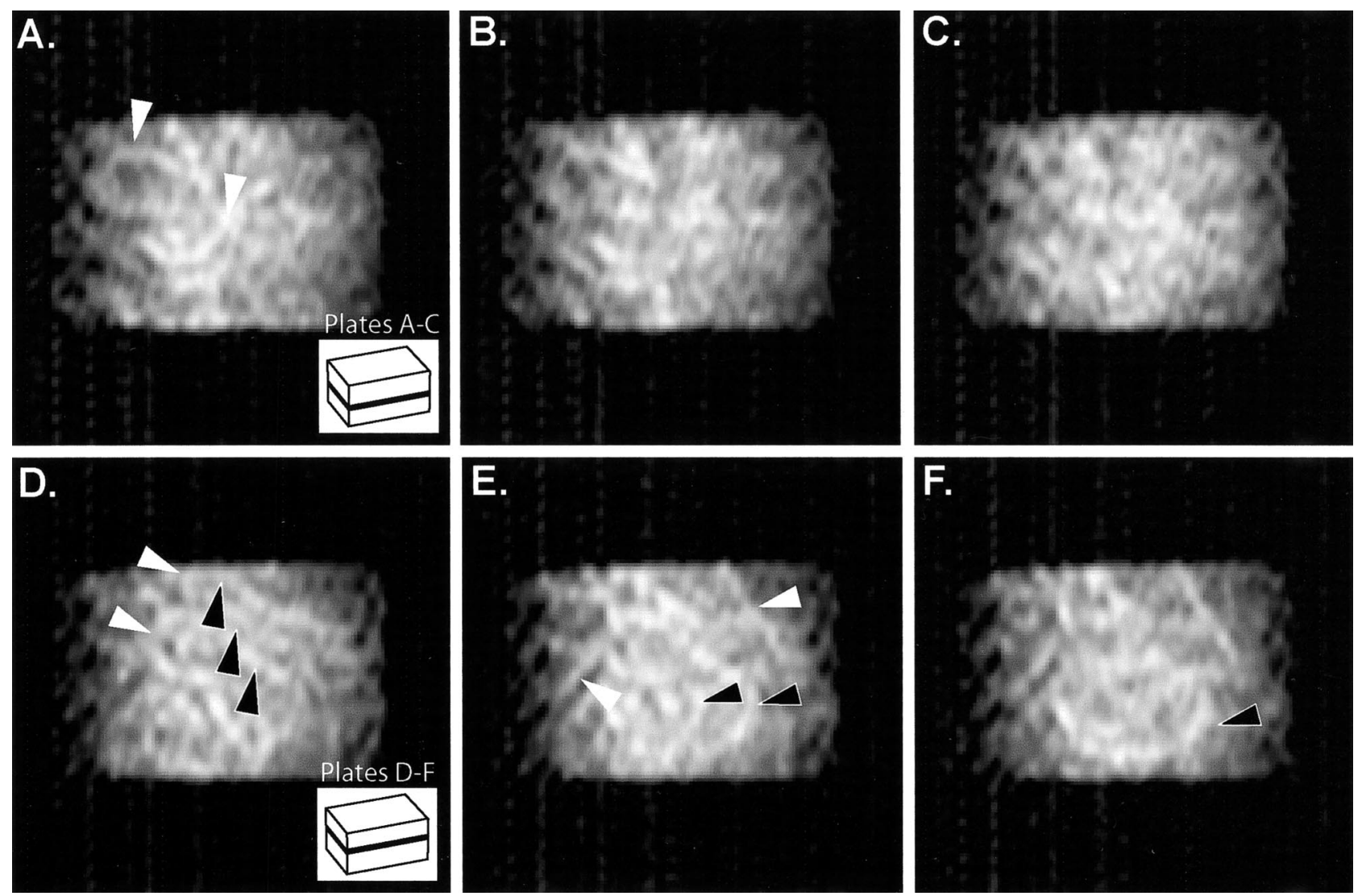

FIG. 4.-MRI horizontal sections that show a range of behaviors between tight meanders (parts A and D), loose meanders (parts E and F), straight burrow segments (parts E and F.), chaotic-appearing random avoidance (random bioturbation present in all images), and unburrowed media (parts B and C). A) Tight meander indicated by white arrows. B-C) Lightly bioturbated media illustrating the complexity of the MRI-resolved fabric. The general mottled texture in parts B and C are the result of the image showing primarily unburrowed rock out of the zone of intense reworking. D) Burrow meanders are visible (white arrows), note the en-echelon nature of concordant burrows, which demonstrates phobotaxis (black arrows). E-F) Straight (white arrows) to sinuous burrows (black arrows) are shown.

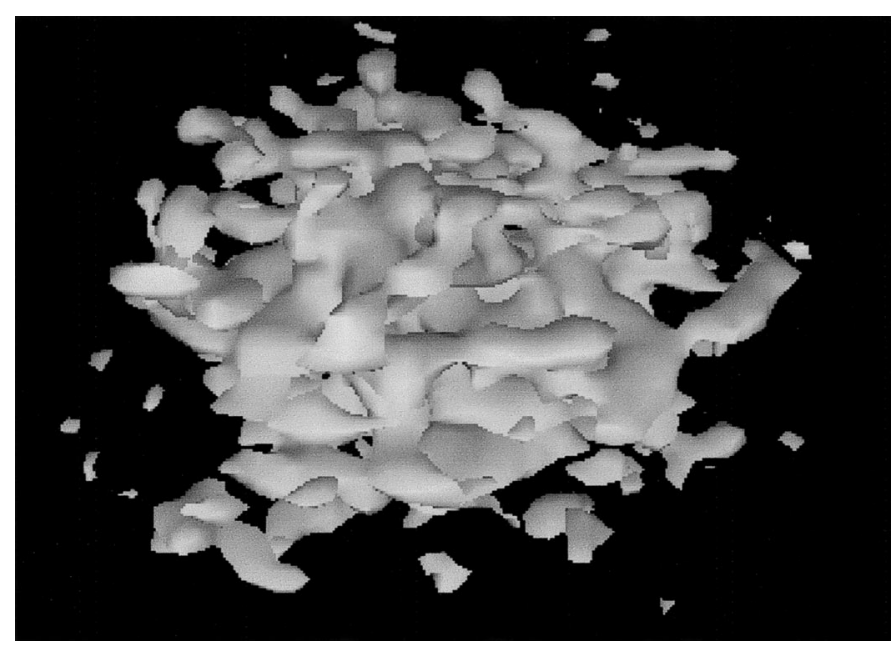

FIG. 5.-Three-dimensional surface rendering of the higher MRI signal. The signal roughly correlates to porosity zones of $12 \%$ and higher. Note the complexity and interwoven nature shown by the burrow interrelationships. The burrows appear to occupy much of the volumetric space available in the sediment block. though this behavior was followed through various larval growth stages, the study did not encompass opheliid adulthood.

The general segregation of chert and plagioclase from quartz has notable significance where considering the distribution of porosity in the matrix. Increased compaction is associated with the mechanically less competent chert and plagioclase grains, resulting in a net reduction of the intergranular porosity (Fig. 2A). Mechanical compaction has also reduced the pore throat size (Fig. 2A), presumably lowering the permeability of the unburrowed sandstone. This suggests that Macaronichnusburrowed sands comprise discrete, complexly juxtaposed zones of differing matrix and burrow porosity-permeability and can be thought of as a dual porosity-permeability flow, or storage, medium. Fractured media are a more extreme example of this, wherein the fractures and the rock may have vastly differing flow properties, but fluids passing through them interact with both flow fields. In the case of the burrowed media, the coincidence of known higher porosity zones and high MR signal (Figs. 3, 4, and 5) suggests a strong correlation between both pore size and pore volume, with the MR signal strength. Figure 5 effectively demonstrates the complex distribution of porosity in the sample.

The general morphological characteristics of the burrows indicate that the tracemakers primarily were deposit-feeding along single laminae. Burrow deviation between the laminae reveal that the burrows were emplaced after sediment burial (i.e. are not surface tracks or casts). Although no single foraging strategy can be identified, the absence of interpenetration indicates that the burrowing animals were extremely adverse to previously burrowed sediment (known as phobotaxis). Meanders and coils represent areas where the animal either had room to systematically forage or where animals encountered resource-rich sediment (zones rich in marinederived organic detritus). Given that burrows tend to sweep or tightly meander almost parallel to each other, it is most plausible that patchy resource in the sediment explains these behaviors. In some horizons, no discernible burrow patterns are observed. Chaotic bioturbation may result from exceptionally high population densities where burrowing organisms actively avoid previously harvested substrate (random avoidance; cf. Saunders and Pemberton 1990). 

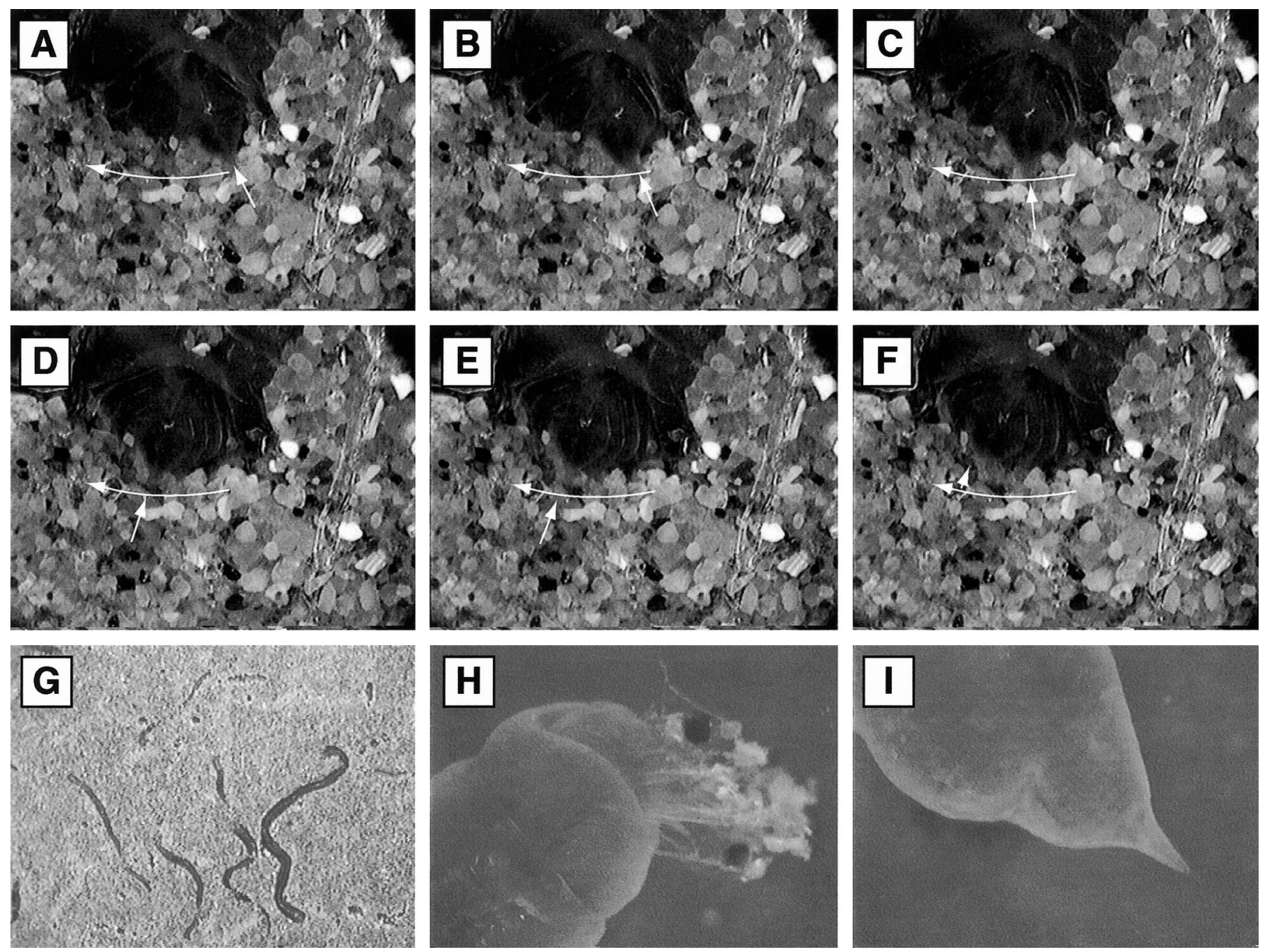

FIg. 6.-A-F) Time-lapse video (approximately 1/10 second lapsed between frames) of the action of the posterior of a modern worm (Euzonis) as it backfills its burrow (small white arrow indicates the location of the worms "tail" or pygidium). The sweeping motion is shown with the longer, curved arrow. These worms are known modern analogs for Macaronichnus trace fossils. G) Euzonis burrowing through sediment against aquarium glass. Note the slightly lighter-appearing sediment trails. H) The posterior end of the worm Euzonis. The anus is swept back and forth as the animal progresses through the sediment. I) The proboscis on the anterior end of the worm. This appendage is used to probe the sediment before grains are taken in by the mouth parts that are beneath this structure. All frames courtesy of Mike Ranger, Tom Saunders, and Kevin Brett.

\section{Discussion of Porosity Distribution}

The dual porosity-permeability system has notable implications where applied to resource quality in porous media. It has been previously demonstrated that burrow systems that are characterized by biogenically induced textural heterogeneity can directly influence the quality of a reservoir or aquifer (Gingras et al. 1999). Conceptually, these fabrics have some of the flow characteristics that fracture systems exhibit. Several of the considerations pertinent to fluid production from sedimentary rocks are: (1) diffusion into and out of the unburrowed media affect the rate at which a fluid or contaminant might be removed from the rock; (2) fluids in higherpermeability conduits can be cut off from the main flow channels, reducing the overall recoverable resource in a reservoir; (3) the net permeability and porosity are a non linear function of the matrix and burrow properties (Gingras et al. 1999); (4) understanding these systems in detail requires appropriate modeling and laboratory experimentation; and (5) flow networks might control later diagenetic processes, such as cement precipitation and dissolution.

The preceding points emphasize that much research needs to be done to demonstrate the significance of Macaronichnus burrowed and similarly burrowed strata. Although they fall outside the scope of this paper, some of the techniques used herein will be useful in characterizing similarly chaotic sediments.

\section{CONCLUSIONS}

Magnetic resonance imaging techniques have helped to characterize the threedimensional geometry of Macaronichnus segregatis in a small sample. Burrow morphologies included straight, sinuous, tightly meandered, and spiraled geometries. Petrographic data confirm the actively back-filled nature of the trace fossil and the likelihood that grain selection and passive sorting by the tracemaker generates the observed fabric. Changes in burrow morphology represent responses primarily to population density and patchy resource distribution. Chaotic fabrics represent high population densities, and small meanders and spirals probably indicate early arrival in a resource-rich substrate.

Perhaps more importantly, the technique was useful for mapping the distribution of porous zones in the sample. It must be stressed that MR images represent complex composites of nuclear spin density, MR relaxation times, and pore size. In this application, stronger MR signals were calibrated to known porous zones by integrating petrological data with MR data. By combining these data, it is possible to gain an understanding of how the grain arrangement evolved. Furthermore, the impact of the sedimentary texture on the reservoir- or aquifer-quality of similar sedimentary rocks can be considered.

Future research should focus on the dual porosity-permeability nature of similar 
fabrics. These studies might be conducted at several scales. The images provided herein show the potential of MRI research in sedimentary studies.

\section{ACKNOWLEDGMENTS}

Video-frames were donated by Mike Ranger, Tom Saunders, and Kevin Brett. The MR images were made in the MRI laboratory at the University of New Brunswick. The operation of this lab is made possible through a major facilities grant from the Natural Science and Engineering Research Council (NSERC) of Canada an NSERC operating research grant, and Steacie Fellowship (2000-2002) to BJB Sampling and petrographic work was funded through an NSERC research grant to MKG. The manuscript was improved through the efforts of JSR reviewers Patrick Jacobs, Duncan McIlroy, and Associate Editor Richard Worden.

\section{REFERENCES}

Attard, J.J., McDonald, P.J., Roberts, S.P., and Taylor, T. 1994, Solid state NMR imaging of irreducible water in reservoir cores for spatially resolved pore surface relaxation estimation: Magnetic Resonance Imaging, v. 12, p. 355-359.

BencsiK, M. AND Ramanathan, C., 2001, Direct measurement of porous media local hydrodynamical permeability using gas MRI: Magnetic Resonance Imaging, v. 19, p. 379-383.

Brown, M.A., and SemelKa, R.C., 1999, MRI: Basic Principles and Practices: John Wiley \& Sons, $224 \mathrm{p}$.

Clifton, H.E., and Thompson, J.K., 1978, Macaronichnus segregatus: A feeding structure of shallow marine polychaetes: Journal of Sedimentary Petrology, v. 48, p. 1293-1302.

Dотт, R.H., 1964, Wacke, graywacke and matrix; what approach to immature sandstone classification?: Journal of Sedimentary Petrology, v. 34. p. 625-632.

Gingras, M.K., MacEachern, J.A., And Pemberton, S.G., 1997, A comparative analysis of the ichnology of wave- and river-dominated allomembers of the Upper Cretaceous Dunvegan Formation: Bulletin of Canadian Petroleum Geology, v. 46, p. 51-73.

Gingras, M.K., Pemberton, S.G., Mendoza, C., and Henk, F., 1999, Modeling fluid flow in trace fossils: assessing the anisotropic permeability of Glossifungites surfaces: Petroleum Geoscience. v. 5, p. 349-357.

Guilfoyle, D.N., Issa, B., ANd Mansfield, P., 1996, Rapid volumetric NMR imaging of fluids in porous solids using a 3D $\pi$-EPI (PEPI) hybrid: Journal of Magnetic Resonance, v. A119, p 151-156.

HowARD, J.J., 1998, Quantitative estimates of porous media wettability from proton NMR measurements: Magnetic Resonance Imaging, v. 16, p. 529-533.

Howard, J.J., AND KeNYon, W.E., 1992, Determination of pore size distribution in sedimentary rocks by proton nuclear magnetic resonance: Marine and Petroleum Geology, v. 9, p. 139145.

KenYon, W.E., 1992, Nuclear magnetic resonance as a petrophysical measurement: Nuclear Geophysics, v. 2. p. 153-171.

Lavigne, J.M., 1999, Sedimentology, stratigraphy, and ichnology of The Horseshoe Canyon Formation [unpublished Master's thesis]: Edmonton, University of Alberta, $146 \mathrm{p}$
MacEachern, J.A., and Pemberton, S.G., 1992, An integrated ichnological-sedimentological model of Cretaceous shoreface successions and shoreface variability in the Western Interior Seaway of North America: in Pemberton, S.G., ed., Applications of Ichnology to Petroleum Exploration-a core workshop: SEPM, Core Workshop 17, p. 57-84.

MatwiYoff, N.A., 1990, Magnetic Resonance Workbook: New York, Raven Press, 120 p.

Miner, B.G., Sanford, E., Strathmann, R.R., Pernet, B., and Emlet, R.B., 1999, Functional and evolutionary implications of opposed bands, big mouths, and extensive oral ciliation in larval opheliids and echiurids (Annelida): Biological Bulletin Woods Hole, v. 197, p. 14-25.

NARA, M., 1994, What is the producer of "trace fossil of Excirolana chiltoni"-Tracemaking mechanism of Macaronichnus segregatus (in Japanese): Fossils, v. 56, p. 9-20.

Rack, F.R., Balcom, B.J., MacGregor, R.P., and Armstrong, R.L., 1998, Magnetic resonance imaging of the Lake Agassiz-Lake Winnipeg transition: Journal of Paleolimnology, v. 19, p. 255-264.

Saunders, T.D.A., and Pemberton, S.G., 1986, Trace fossils and sedimentology of the Apaloosa Sandstone: Bearpaw-Horseshoe Canyon Formation transition, Dorothy, Alberta: Canadian Society of Petroleum Geologists, Field Trip Guide Book, 117 p.

Saunders, T.D.A., and Pemberton, S.G., 1990, On the palaeoecological significance of the trace fossil Macaronichnus (abstract), in $13^{\text {th }}$ International Sedimentological Congress, In ternational Association of Sedimentologists, Nottingham, England.

Received 8 March 2001; accepted 29 December 2001.

\section{APPENDIX 1}

Image Acquisition Parameters:

1. Conventional spin echo technique.

2. Primary phase gradient:- 4 to $4 \mathrm{G} / \mathrm{cm}$ in 127 steps (for 128 different values).

3. Secondary phase encode gradient stepped through 64 values with increments similar to the primary phase encode gradient; this was done with zero infilling to 128 points.

4. Final data matrix: $128^{3}$ data points.

5. Acquisition time for four-signal-average was 55 minutes.

6 . The image signal to noise ratio was $\approx 16$; the ratio of image contrast to noise was $\approx 9$.

7. MR echo time of $1.5 \mathrm{~ms}$; repetition time of $100 \mathrm{~ms}$.

8. Images reconstructed with a 3-D Fast Fourier Transform.

Equipment:

1. General Electric (GE NMR Instruments, Fremont, CA) CSI II spectrometer in an Oxford (Oxford Instruments, Oxford, England) 2.0 Tesla, $31 \mathrm{~cm}$ i.d. horizontal bore superconducting magnet (at ambient temperature)

2. General Electric Acustar $15 \mathrm{~cm}$ i.d. gradient set (maximum gradient strength 10 $\mathrm{G} / \mathrm{cm}$ ) powered by Techron 8608 amplifiers.

3. The radio frequency (r.f.) probe was a custom, single channel, $57 \mathrm{~mm}$ i.d., 48 rung birdcage

4. The GE spectrometer used a built-in 100-Watt amplifier. 